\title{
Limit Cycles of Lorenz System with Hopf Bifurcation
}

\section{Azad.I.Amen}

College of Basic Education

University of Salahaddin

Received on: 09/07/2006
Rizgar .H. Salih

College of science

University of Sulaimani

Accepted on: 05/03/2007

\section{ABASTRACT}

We prove that near the bifurcation point unstable limit cycle arises from the Lorenz system. In the analysis, we use the method of local bifurcation theory, especially the center manifold and the normal form theorem. A computer algebra system using Maple to derive all the formulas and verify the results presented in this paper.

Keywords: Lorenz system, limit cycle, center manifold and normal form, Hopf bifurcation.

الارات الغائية لنظام Lorenz بتفرع Hopf

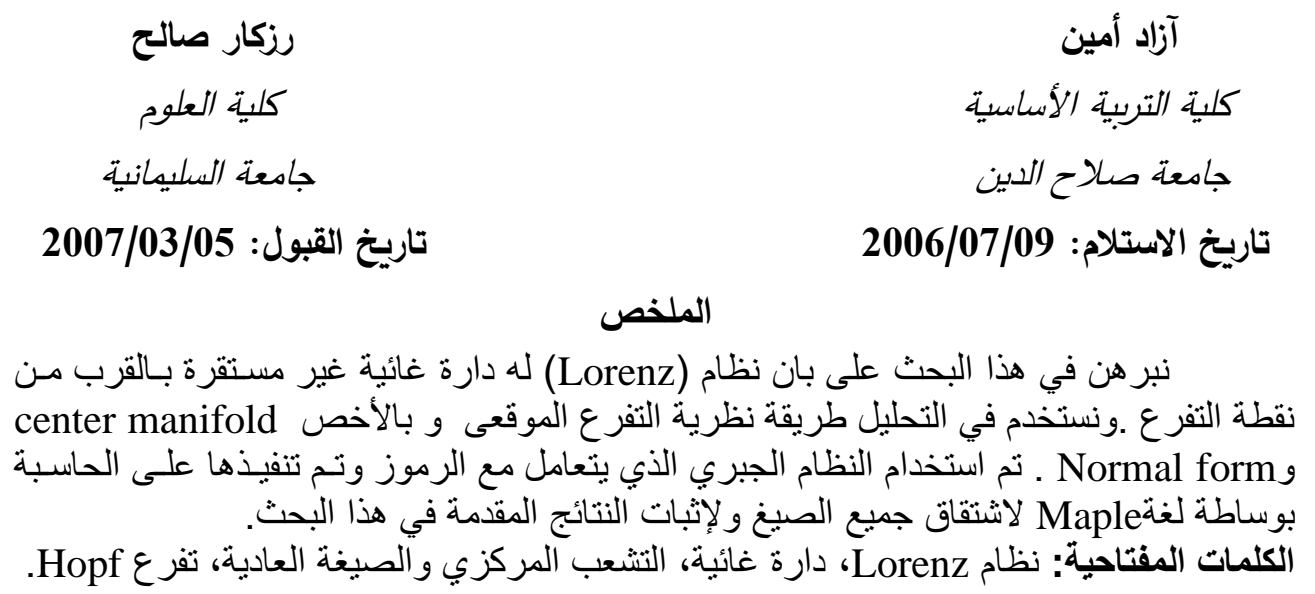

\section{Introduction}

The following non-linear system of differential equation

$$
\begin{aligned}
& \dot{x}=\sigma(y-x) \\
& \dot{y}=r x-y-x z \\
& \dot{z}=-\beta z+x y
\end{aligned}
$$

was introduced in 1963 by Edward Lorenz [6] ( known as Lorenz system), where the three parameters $\sigma, \beta$ and $r$ are positive. These equations also arise in studies of convection and instability in Planetary atmospheres, models of Lasers and dynamos etc. Lorenz studied the system when $\sigma=10, \beta$ $=\frac{8}{3}$ (see[7]). Good notes on Lorenz system can be found in [14]. In general, Lorenz system can't be integrable and it is difficult to find analytical solution for system (1) in the three dimension parameters space, but special 
cases for Lorenz system are studied before studying periodic solutions [9].The behavior of the system (1) is quiet complex.

From the historical point of view the search on the existence of periodic solution plays a fundamental role in the development of qualitative study of dynamical system. There are many methods for locating the periodic solution,for example the Poincare return map, Melnikov integral, degree theory and bifurcation theory. In this paper, we shall use Hopf bifurcation theorem for finding the limit cycle (isolated closed orbits) of the Lorenz system.

The paper consists of four sections. In section two we start by recalling the well know center manifold and normal form theorem for the general equilibrium point. Section three gives a detailed analysis of the Hopf bifurcation, using the method of local bifurcation theory, especially the center manifold and normal form theorem, it is proved that unstable limit cycle arisesd, also limit cycles corresponding to some special values of $\sigma, \beta$ at the Hopf bifurcation point. The paper ends with a brief discussion of the results. All the results presented obtained and verified using Maple work station ([3], [4], and [7]).

\section{Center Manifold and Normal Form Theorem.}

In this section, we make a brief summary of the techniques we used to reduce the dimension of system (2) by center manifold theorem and normal form theorem to simplify the flow in the center manifold (see[5] , [11]).

The theory of bifurcations of parameterized dynamical system is well known. One consider a vector field

$$
\dot{x}=f_{\mu}(\mathrm{x}) \quad \mu \in \mathrm{R}, \mathrm{x} \in R^{n}
$$

Depending on a parameter $\mu$ the equilibrium of the vector field are those xo, $\mu$ o such thatf $\mu_{\mathrm{o}}(\mathrm{xo})=0$.

Perhaps the most important property of equilibrium is its stability. In the first approximation, which is determined by stability of its liberalized system around xo, $\mu \mathrm{o}$

$$
\dot{x}=\mathrm{D} f_{\mu 0}(\mathrm{xo}) \quad \mu_{\mathrm{O}} \in \mathrm{R}, \mathrm{x}_{\mathrm{O}} \in R^{n}
$$

Where $\mathrm{D} f_{\mu 0}(\mathrm{xo})$ is the Jacobian matrix of $\mathrm{f}$, if all the eignvalues of $\mathrm{D} f_{\mu 0}(\mathrm{xo})$ have negative real parts, the equilibrium point is asymptotically stable. If at least one eignvalues has positive real part, then the equilibrium is unstable [10]. The topological character of the equilibrium can change at a equilibrium value of the parameter, perhaps two branches of equilibrium cross or a branch loses or gains stability. Such a state and parameter is 
called bifurcation point of the parameterized vector field. If a conjugate complex pair crosses the boundary of stability then this bifurcation is called Hopf bifurcation.

Theorem1. (Center manifold theorem for flows) [5]

Let $f$ be a $C^{r}$ vector field on $R^{n}$ vanishing at the origin $(f(0)=0)$ and let $\mathrm{A}=\mathrm{Df}(0)$. Divide the spectrum of $\mathrm{A}$ into three parts, $\sigma_{s}, \sigma_{c}, \sigma_{u}$ with

$\operatorname{Re} \lambda\left\{\begin{array}{l}<0 \text { if } \lambda \in \sigma_{s}, \\ =0 \text { if } \lambda \in \sigma_{c}, \\ >0 \text { if } \lambda \in \sigma_{u} .\end{array}\right.$

Let the (generalized) eignspaces of $\sigma_{s}, \sigma_{c}$, and $\sigma_{u}$ be $E^{s}, E^{c}$, and $E^{u}$, respectively. Then there exists $C^{r}$ stable and unstable invariant manifolds $W^{s}$ and $W^{u}$ tangent to $E^{s}$ and $E^{u}$ at 0 and a $C^{r-1}$ center manifold $W^{c}$ tangent to $E^{c}$ at 0 . The manifolds $W^{s}, W^{u}$, and $W^{c}$ are invariant for the flow of $f$.

The stable and unstable manifolds are unique because its invariant, but $W^{c}$ needs not be since its center is manifold(see[ 2]).

We know that the complete stability and qualitative behavior of an equilibrium point depends on the behavior of the dynamics on the center manifold [2]. We first note that center, stable and unstable manifolds are invariant under the O.D.E. Assume that the O.D.E are

$$
\begin{aligned}
& \dot{x}=A^{c} \mathrm{x}+f_{1}(\mathrm{x}, \mathrm{y}, \mathrm{z}) \\
& \dot{y}=A^{s} \mathrm{y}+f_{2}(\mathrm{x}, \mathrm{y}, \mathrm{z}) \\
& \dot{z}=A^{u} \mathrm{z}+f_{3}(\mathrm{x}, \mathrm{y}, \mathrm{z})
\end{aligned}
$$

Where $A^{c}, A^{s}$ and $A^{u}$ on the blocks are in the canonical form whose diagonals contain the eignvalues with $\operatorname{Re} \lambda=0, \operatorname{Re} \lambda<0$ and $\operatorname{Re} \lambda>0$, respectively, $(\mathrm{x}, \mathrm{y}, \mathrm{z}) \in R^{c} \times R^{s} \times R^{u}, \mathrm{c}=\operatorname{dim} E^{c}$ since the system(4)has c eigenvalues with zero real part ( $E^{c}$ is called the center eigenvector), $\mathrm{s}=\operatorname{dim}$ $E^{s}$ and $\mathrm{u}=\operatorname{dim} E^{u}, f_{1}, f_{2}$ and $f_{3}$ vanish along with their first partial derivatives at the origin.

Let $\mathrm{y}=h_{1}(\mathrm{x})$ be a stable manifold and $\mathrm{z}=h_{2}(\mathrm{x})$ be unstable manifold where $\mathrm{x}$ is on the center manifold then $h_{1}(x)$ and $h_{2}(x)$ satisfy the following equations:

D $h_{1}(\mathrm{x})\left[A^{c} \mathrm{x}+f_{1}\left(\mathrm{x}, h_{1}(\mathrm{x}), h_{2}(\mathrm{x})\right)\right]-A^{s} h_{1}(\mathrm{x})-f_{2}\left(\mathrm{x}, h_{1}(\mathrm{x}), h_{2}(\mathrm{x})\right)=0$

$\mathrm{D} h_{2}(\mathrm{x})\left[A^{c} \mathrm{x}+f_{1}\left(\mathrm{x}, h_{1}(\mathrm{x}), h_{2}(\mathrm{x})\right)\right]-A^{u} h_{2}(\mathrm{x})-f_{3}\left(\mathrm{x}, h_{1}(\mathrm{x}), h_{2}(\mathrm{x})\right)=0$

And the dynamics on center manifold is 


$$
\dot{x}=A^{c} \mathrm{x}+f_{1}\left(\mathrm{x}, h_{1}(\mathrm{x}), h_{2}(\mathrm{x})\right)
$$

because it's the most physically interesting case (among them Lorenz system at the Hopf bifurcation), we assume that the unstable manifold is empty, in this case (4) becomes:

$$
\begin{aligned}
& \dot{x}=A^{c} \mathrm{x}+f_{1}(\mathrm{x}, \mathrm{y}) \\
& \dot{y}=A^{s} \mathrm{y}+f_{2}(\mathrm{x}, \mathrm{y})
\end{aligned}
$$

In this form $E^{c}=\{(\mathrm{x}, \mathrm{y}), \mathrm{y}=0\}$ and $E^{s}=\{(\mathrm{x}, \mathrm{y}), \mathrm{x}=0\}$, note also $f_{1}(0,0)=\mathrm{D} f_{1}(0,0)=0$ and $f_{2}(0,0)=\mathrm{D} f_{2}(0,0)=0$.

The local Center manifold theorem[2] states that :

There exists a $C^{\infty}$, center manifold $W_{l o c}^{c}(0)=\{(\mathrm{x}, \mathrm{y}): \mathrm{y}=\mathrm{h}(\mathrm{x}),|\mathrm{x}|<\delta$, $\mathrm{h}(0)=\operatorname{Dh}(0)=0\}$ Such that the dynamics of (7) restricted to the center manifold are given by

$$
\dot{x}=A^{c} \mathrm{x}+f_{1}(\mathrm{x}, \mathrm{h}(\mathrm{x}))
$$

Theorem 2. [2].

If the origin for(8) is locally asymptotically stable (resp. unstable) then the origin of eq (7) is also locally asymptotically stable (resp. unstable).

Remark: Theorem 2 shows that equation (8) is a good approximation of eq(7).

We now show how $\mathrm{h}(\mathrm{x})$ can be calculated or at least approximated.

Substitute $\mathrm{y}=\mathrm{h}(\mathrm{x})$ in the second component of eq(7) and using the chain rule, we obtain:

$\mathrm{N}(\mathrm{h}(\mathrm{x}))=\mathrm{D} \mathrm{h}(\mathrm{x})\left[A^{c} \mathrm{x}+f_{1}(\mathrm{x}, \mathrm{h}(\mathrm{x}))\right]-A^{s} \mathrm{~h}(\mathrm{x})-f_{2}(\mathrm{x}, \mathrm{h}(\mathrm{x}))=0$

With boundary conditions $\mathrm{h}(0)=\mathrm{D} \mathrm{h}(0)=0$. This differential equation for $\mathrm{h}(\mathrm{x})$ not can be solved exactly in most cases, but its solution can be approximated arbitrarily close, as a Taylor series at $\mathrm{x}=0$.

Theorem 3. [2].

If a function $\phi(x)$ with $\phi(0)=D \phi(0)=0$ can be found such that $\mathrm{N}(\phi(\mathrm{x}))=\mathrm{o}\left(\mid x^{p}\right)$ for some $\mathrm{p}>1$ as $|x| \rightarrow 0$ then it follows that $\mathrm{h}(\mathrm{x})=\phi(\mathrm{x})+$ $\mathrm{o}\left(\mid x^{p}\right)$ as $|x| \rightarrow 0$.

This theorem tells us that we can approximate the center manifold to any degree of approximation by solving the $\mathrm{N}$-equation to the same degree of approximation. A standard approach to analyzing the behavior of the parameterized ordinary differential equation (2) around a bifurcation point is to treat the parameter as an additional state variable with dynamics $\dot{\mu}=0$ and to compute the center manifold of the extended dynamics through the 
bifurcation point and the dynamics restricted to this manifold [5]. The center manifold is an invariant manifold of the differential equation which is tangent at the bifurcation to the eignspase of the neutrally stable eigenvectors. In practice, one does not compute the center manifold and its dynamics exactly, in most cases of interest, an approximation of degree two or three suffices.

For simplifying the flow in the center manifold the normal form theorem is used in order to remove all non necessary terms up to a certain order while retaining the right qualitative behavior of the system at the bifurcation point. The result of this simplification is the so called normal form of the flow. Normal form theory has been widely used in the study of non linear vector field, in order to simplify the analysis of the original system,it provides a convenient tool to transform a given system to an equivalent system whose dynamical behavior is easier to analyze ([16], and [18]).

If the system (2) has Hopf bifurcation at $\mathrm{x}_{\mathrm{O}}, \mu_{\mathrm{O}}$ then $\mathrm{D} f_{\mu 0}\left(\mathrm{x}_{\mathrm{O}}\right)$ has a simple pair of pure imaginary eignvalues, $\pm i \omega, \omega>0$ and no other eigenvalues with zero real part. By smooth changes of coordinates the Taylor series of degree three for the general problem can be brought to the following form ([5], [8], and [17])

$$
\begin{aligned}
& \dot{x}=\left(d \mu+a\left(x^{2}+y^{2}\right)\right) x-\left(\omega+c \mu+b\left(x^{2}+y^{2}\right)\right) y \\
& \dot{y}=\left(\omega+c \mu+b\left(x^{2}+y^{2}\right)\right) x+\left(d \mu+a\left(x^{2}+y^{2}\right)\right) y
\end{aligned}
$$

This is expressed in polar coordinates :

$$
\begin{aligned}
& \dot{\rho}=\left(d \mu+a \rho^{2}\right) \rho \\
& \dot{\theta}=\left(\omega+c \mu+b \rho^{2}\right)
\end{aligned}
$$

Since the $\dot{\rho}$ in equation (9) separates from $\theta$, we see that there are periodic orbits of (8) which are circles $\rho=$ const., obtained from the nonzero solution of $\dot{\rho}$ in equation (9); if $\mathrm{a} \neq 0$ and $\mathrm{d} \neq 0$ these solutions lie along the parabola $\mu=-\frac{a \rho^{2}}{d}$. This implies that the surface of periodic orbits has a quadratic tangency with its tangent plane $\mu=0$ in $R^{2} \times R$. The content of the Hopf bifurcation theorem is that the qualitative properties of equation (8) near the origin remains unchanged if higher order terms are added to the system.

\section{Theorem 4 (Hopf Bifurcation theorem) [5]}

Suppose that the system $\dot{x}=f_{\mu}(\mathrm{x}), \mu \in \mathrm{R}, \mathrm{x} \in R^{n}$ has equilibrium $\left(\mathrm{x}_{\mathrm{O}}\right.$, $\mu_{\mathrm{o}}$ ) at which the following properties are satisfied: 
(H1) $\mathrm{D} f_{\mu 0}$ (xo) has a simple pair of pure imaginary eignvalues and no other eignvalues with zero real parts.

(H2) $\left.\frac{d}{d \mu}\left(\operatorname{Re}\left(\lambda_{2 / 3}(\mu)\right)\right)\right|_{\mu=\mu o}=\mathrm{d} \neq 0$,

where $\operatorname{Re}\left(\lambda_{2 / 3}(\mu)\right)$ denotes the real part of $\lambda$ which is a smooth function of $\mu$. Then there exists a unique three-dimensional center manifold passing through $\left(\mathrm{x}_{\mathrm{O}}, \mu_{\mathrm{O}}\right)$ in $R^{n} \times R$ and a smooth system of coordinates (preserving the planes $\mu=$ const.) for which the Taylor expansion of degree three on the center manifold is given by (8). If a $\neq 0$, there is a surface of periodic solution in the center manifold which has quadratic tangency with the eigenspace of $\lambda(\mu \mathrm{o}), \bar{\lambda}(\mu \mathrm{o})$ agreeing to second order with the parabolic $\mu=-$ $\frac{a}{d}\left(x^{2}+y^{2}\right)$. If $a<0$, then this periodic solution is stable limit cycles, while if a $>0$, the periodic solutions are repelling (unstable limit cycles).

3. Stability and Hopf Bifurcation analysis

Now Our aim is to apply the method described in section two, to system (1) the goal of analyzing the Lorenz system at the equilibrium points and analyzes the Hopf bifurcation theorem of system (1),calculations have been done with the symbolic processor Maple.

\section{1-Equilibrium points and Stability.}

From the definition of equilibrium point, it is easy to verify that, when $r \leq 1$ system (1) has only one-equilibrium point, which is the origin, but when $\mathrm{r}>1$ it has three equilibrium points :

$\mathrm{O}=(0,0,0), A_{\mp}=(\mp \sqrt{\beta(r-1)}, \mp \sqrt{\beta(r-1)}, \mathrm{r}-1)$

Let $\lambda^{3}-T \lambda^{2}-K \lambda-\mathrm{D}=0$ be the characteristic polynomial for a three-component system, where T,D indicate the trace and determinant rest, then a Hopf bifurcation takes place of the transit through the surface $\mathrm{TK}+\mathrm{D}=0$ if $\mathrm{T}, \mathrm{K}, \mathrm{D}<0$ [1] and [13]).

This condition is a necessary condition for a Hopf bifurcation(

If $\mathrm{T}, \mathrm{K}, \mathrm{D}<0$ and $\mathrm{TK}+\mathrm{D}>0$

then all eignvalues has negative real part, this is the necessary and sufficient condition which is required for stability of equilibrium points of a three dimensional system and are called Routh-Hurwitz criterion[12]. 
The Jacobian matrix of system $(1)$ at $\mathrm{O}=(0,0,0)$ is:

$J_{0}=\left[\begin{array}{ccc}-\sigma & \sigma & 0 \\ r & -1 & 0 \\ 0 & 0 & -\beta\end{array}\right]$

characteristic equation of $J_{0}$ is:

$$
\begin{array}{ll} 
& \lambda^{3}+(\sigma+\beta+1) \lambda^{2}+(\beta(\sigma+1)+\sigma(1-r)) \lambda+\beta \sigma(1-r)=0 \\
\text { Then } \mathrm{T}=-(\sigma+\beta+1) & \mathrm{K}=-\beta(\sigma+1)-\sigma(1-\mathrm{r}) \\
\mathrm{D}=-\beta \sigma(1-\mathrm{r}) & \\
& T K+D=\beta^{2}(\sigma+1)+\beta\left(\sigma^{2}+1\right)+2 \beta \sigma+\sigma(\sigma+1)-r \sigma(\sigma+1)>0 \quad \text { if } r<1
\end{array}
$$

It follows from the Routh-Hurwitz criterion with (14-17) that the origin is stable within the range $0<\mathrm{r}<1$.

The Jacobian matrix of system (1) at $A_{+}$is:

$$
J_{1}=\left[\begin{array}{ccc}
-\sigma & \sigma & 0 \\
1 & -1 & -\sqrt{\beta(r-1)} \\
\sqrt{\beta(r-1)} & \sqrt{\beta(r-1)} & -\beta
\end{array}\right]
$$

The characteristic equation of $J_{1}$ is:

$$
\lambda^{3}+(\sigma+\beta+1) \lambda^{2}+\beta(\sigma+r) \lambda+2 \beta \sigma(r-1)=0
$$

Then

$$
\begin{aligned}
& \mathrm{T}=-(\sigma+\beta+1) \\
& \mathrm{K}=-\beta(\sigma+\mathrm{r}) \\
& \mathrm{D}=-2 \beta \sigma(\mathrm{r}-1) \\
& T K+D=\beta \sigma(\sigma+\beta+3)-r \beta(\sigma-\beta-1)>0 \quad \text { if } r<r_{0}=\frac{\sigma(\sigma+\beta+3)}{\sigma-\beta-1}
\end{aligned}
$$

it follows from the Routh-Hurwitz criterion with (20-23) that $A_{+}$is stable within the range

$$
\left.1<\mathrm{r}<r_{0}=\frac{\sigma(\sigma+\beta+3)}{\sigma-\beta-1} \text { when } \sigma>\beta_{+} \quad \text { (see figure } 1\right) .
$$

Since at $r=1$ change of stability occurs, at which $T K+D \neq 0$ then a Hopf bifurcation cannot appear of the Lorenz system at the origin. But also at $\mathrm{r}=$ $r_{0}=\frac{\sigma(\sigma+\beta+3)}{\sigma-\beta-1}$ change of stability occurs, at which $\mathrm{TK}+\mathrm{D}=0$ then a Hopf bifurcation appears of the Lorenz system at the $A_{+}$.

Remark: the bifurcation at $\mathrm{r}=1$ is called Pitch fork bifurcation [6].

\section{2 - Hopf Bifurcation}

If the parameters satisfy the condition $r=r_{0}=\frac{\sigma(\sigma+\beta+3)}{\sigma-\beta-1}$, then the matrix $J_{1}$ has one negative and two purely imaginary eignvalues: 
$\lambda_{1}=-(\sigma+\beta+1)$
$\lambda_{2 / 3}= \pm i \omega$ where $\omega=\sqrt{\frac{2 \beta \sigma(\sigma+1)}{\sigma-\beta-1}}$

So the first condition for a Hopf bifurcation (theorem 4) is fulfilled. Nevertheless for applying the Hopf bifurcation theorem a second condition must be fulfilled.The conjugate of complex eignvalue which is imaginary at the bifurcation point, $\lambda_{2 / 3}(r)$ must, if the parameter $r$ is varied and the other parameters are fixed, cross the imaginary axes in the simple way.

$$
\frac{d}{d r}\left(\left.\operatorname{Re}\left(\lambda_{2 / 3}(r)\right)\right|_{r=r o}=\mathrm{d} \neq 0\right.
$$

where $\operatorname{Re} \lambda_{2 / 3}((\mathrm{r}))$ denotes the real part of $\lambda$ which are smooth functions of $r$. We now calculate $d$ without solving (19) explicitly. Let $\lambda_{2}=u_{1}+$ $\mathrm{i} v_{1}, \lambda_{3}=\bar{\lambda}_{2}=u_{1}$-i $v_{1}$ and $\lambda_{1}$ be eignvalues of $J_{1}$. As $J_{1}$ has two non-zero pure imaginary eignvalues when $\mathrm{r}=r_{0}$, it follows that for $\mathrm{r}$ near $r_{0}$ two of the eignvalues will be complex conjugates. $\lambda_{2}, \bar{\lambda}_{2}$ and $\lambda_{1}$ satisfy $x^{3}-\left(2 u_{1}+\lambda_{1}\right) x^{2}+\left(\left|\lambda_{2}\right|^{2}+2 u_{1} \lambda_{1}\right) x-\left|\lambda_{2}\right|^{2} \lambda_{1}=0$

Equating coefficients with equation(19) resulting

$$
\begin{aligned}
& -(\sigma+\beta+1)=2 u_{1}+\lambda 1 \\
& -2 \beta \sigma(\mathrm{r}-1)=\left|\lambda_{2}\right|^{2} \lambda_{1} \\
& \beta(\sigma+\mathrm{r})=\left|\lambda_{2}\right|^{2}+2 u_{1} \lambda_{1}
\end{aligned}
$$

Thus

$$
\frac{2 \beta \sigma(r-1)}{\sigma+\beta+1+2 u_{1}}-2 u_{1}\left(\sigma+\beta+1+2 u_{1}\right)=\beta(\sigma+\mathrm{r})
$$

Implicitly differentiating $u_{1}=u_{1}(\mathrm{r})$, we obtain:

$$
\dot{u}_{1}=\frac{\beta\left(\sigma+\beta+1+2 u_{1}\right)\left(\sigma-\beta-1-2 u_{1}\right)}{4 \beta \sigma(r-1)+2\left(\sigma+\beta+1+4 u_{1}\right)\left(\sigma+\beta+1+2 u_{1}\right)^{2}}
$$

At $\mathrm{r}=r_{0}$ where $\operatorname{Re}\left(\lambda_{2}\right)=u_{1}=0$, after some calculation we obtain:

$$
\dot{u}_{1}\left(r_{0}\right)=\frac{\beta(\sigma-\beta-1)^{2}}{4 \beta \sigma(\sigma+1)+2(\sigma+\beta+1)^{2}(\sigma-\beta-1)}
$$

Since $\sigma>\beta+1$, we have $\dot{u}_{1}\left(r_{0}\right)=\mathrm{d}>0$.

Thus, also the second condition for a Hopf bifurcation is fulfilled and the Hopf bifurcation theorem holds

Remark: Because system (1) is invariant under the transformation (x, y, z) $\rightarrow$ (-x,-y, z), one only needs to consider the stability of system (1) at $A_{+}$. 
We now analyse the Hopf bifurcation of system (1) in detail. At first, we are given an expression for the flow in the center manifold $W^{c}$ at the bifurcation point which is two-dimensional $\left(W^{c}\right.$ has some dimension as the eignspase of the conjugate complex eignvalue with zero real part). Before using the center manifold theorem one has to translate the equilibrium point $A_{+}$to in the origin. In the new coordinates.

$$
\mathrm{x}-\sqrt{\beta(r-1)} \rightarrow y_{1}, \quad \mathrm{y}-\sqrt{\beta(r-1)} \rightarrow y_{2}, \quad \mathrm{z}-(\mathrm{r}-1) \rightarrow y_{3}
$$

System (1) reads:

$$
\begin{aligned}
& \dot{y}_{1}=\sigma\left(y_{2}-y_{1}\right) \\
& \dot{y}_{2}=y_{1}-y_{1} y_{3}-y_{2}-\sqrt{\beta(r-1)} y_{3} \\
& \dot{y}_{3}=y_{1} y_{2}+\sqrt{\beta(r-1)}\left(y_{1}+y_{2}\right)-\beta y_{3}
\end{aligned}
$$

Using the eigenvectors as a base for a new coordinate system, we set

$$
\left[\begin{array}{l}
y_{1} \\
y_{2} \\
y_{3}
\end{array}\right]=P\left[\begin{array}{l}
u \\
v \\
w
\end{array}\right]
$$

where

$$
\begin{aligned}
& \mathrm{P}=\left[\begin{array}{ccc}
-\frac{\sigma(\sigma+1)}{e(\sigma-\beta-1)} & \frac{e(\omega \sigma-\sigma(\sigma+1) i)}{\omega^{3}+\omega(\sigma+1)^{2}} & \frac{e(\omega \sigma+\sigma(\sigma+1) i)}{\omega^{3}+\omega(\sigma+1)^{2}} \\
\frac{(\beta+1)(\sigma+1)}{e(\sigma-\beta-1)} & \frac{e\left(-\left(\omega-\left(\omega^{2}+\sigma(\sigma+1)\right) i\right)\right.}{\omega^{3}+\omega(\sigma+1)^{2}} & \frac{e\left(-\left(\omega+\left(\omega^{2}+\sigma(\sigma+1)\right) i\right)\right.}{\omega^{3}+\omega(\sigma+1)^{2}} \\
1 & 1
\end{array}\right] \\
& {\left[\begin{array}{l}
\dot{u} \\
\mathrm{v} \\
\dot{w}
\end{array}\right]\left(\begin{array}{ccc}
-(\sigma+\beta+1) & 0 & 0 \\
0 & -\mathrm{i} \omega & 0 \\
0 & 0 & \mathrm{i} \omega
\end{array}\right)\left[\begin{array}{c}
\mathrm{u} \\
\mathrm{v} \\
\mathrm{w}
\end{array}\right]+\left(\begin{array}{c}
a_{1} u^{2}+a_{2} v^{2}+\bar{a}_{2} w^{2}+a_{3} u v+\bar{a}_{3} u w+a_{4} v w \\
e_{1} u^{2}+e_{2} v^{2}+e_{3} w^{2}+e_{4} u v+e_{5} u w+e_{6} v w \\
\bar{e}_{1} u^{2}+\bar{e}_{3} v^{2}+\bar{e}_{2} w^{2}+\bar{e}_{5} u v+\bar{e}_{4} u w+\bar{e}_{6} v w
\end{array}\right)}
\end{aligned}
$$

With: $\quad a_{1}=\frac{\sigma(\sigma+1)^{2}(\sigma-2-2 \beta)}{A(\sigma-\beta-1)}$

$$
\begin{aligned}
& a_{2}=\frac{-(\sigma-\beta-1)\left[\sigma \beta\left(3 \sigma^{2}-3+3 \beta+\sigma \beta\right)-i \omega\left(\sigma^{3}-\sigma^{2}-2 \sigma^{2} \beta-\sigma+1-\sigma \beta^{2}+2 \sigma \beta-\beta^{2}\right)\right]}{2 A(\sigma-1)^{2}} \\
& a_{3}=\frac{-\sigma(\sigma+1)\left[\omega\left(-\sigma^{2}+1-\beta(3+2 \beta-\sigma)\right)(\sigma-\beta-1)+i \beta(\sigma+1)\left(-2 \sigma^{2}-2 \beta^{2}+4 \sigma-4 \beta+2 \sigma \beta-2\right)\right]}{\omega A(\sigma-1)(\sigma-\beta-1)}
\end{aligned}
$$




$$
\begin{aligned}
& a_{4}=\frac{-\sigma \beta(\sigma-\beta-1)(\sigma+1-\beta)}{A(\sigma-1)} \\
& e_{1}=\frac{\sigma A_{2}\left[e(\sigma-\beta-1) d_{2}-(\sigma+1)(\beta+1) d_{3}\right]}{\beta(\sigma-\beta-1)(\sigma+\beta+1)} \\
& e_{2}=\frac{A_{2}(\sigma-\beta-1)\left[-2 e \sigma \beta(\sigma-1) d_{2}-\sigma \beta(4 \beta+(\sigma-1)(\sigma+\beta+1)) d_{3}\right.}{2 \beta(\sigma-1)^{2}(\sigma+1)(\sigma+\beta+1)} \\
& -\frac{i \omega\left(-e(\sigma-1)(\sigma-\beta-1) d_{2}+\beta(2 \sigma \beta+(\sigma-1)(\sigma-\beta-1)) d_{3}\right]}{2 \beta(\sigma-1)^{2}(\sigma+1)(\sigma+\beta+1)} \\
& e_{3}=\frac{A_{2}(\sigma-\beta-1)\left[-2 e \sigma \beta(\sigma-1) d_{2}-\sigma \beta(4 \beta+(\sigma-1)(\sigma+\beta+1)) d_{3}\right.}{2 \beta(\sigma-1)^{2}(\sigma+1)(\sigma+\beta+1)} \\
& +\frac{+i \omega\left(-e(\sigma-1)(\sigma-\beta-1) d_{2}+\beta(2 \sigma \beta+(\sigma-1)(\sigma-\beta-1)) d_{3}\right]}{2 \beta(\sigma-1)^{2}(\sigma+1)(\sigma+\beta+1)} \\
& e_{4}=\frac{A_{2} \sigma\left[\omega(\sigma+\beta+1)\left(\sigma^{2}-1-\beta(\sigma-\beta-1)\right) d_{2}+e \omega(\sigma-\beta-1)(2+\beta) d_{3}\right.}{e \omega(\sigma-1)(\sigma-\beta-1)(\sigma+\beta+1)} \\
& +\frac{\left.+i\left(e(\sigma+1)\left(2 \sigma \beta+(\sigma-\beta-1)^{2}\right) d_{3}+\beta(\sigma-\beta-1)(\sigma+\beta+1)(\sigma+1)\right) d_{2}\right]}{e \omega(\sigma-1)(\sigma-\beta-1)(\sigma+\beta+1)} \\
& e_{5}=\frac{A_{2} \sigma\left[\omega(\sigma+\beta+1)\left(\sigma^{2}-1-\beta(\sigma-\beta-1)\right) d_{2}+e \omega(\sigma-\beta-1)(2+\beta) d_{3}\right.}{e \omega(\sigma-1)(\sigma-\beta-1)(\sigma+\beta+1)} \\
& -\frac{\left.i\left(e(\sigma+1)\left(2 \sigma \beta+(\sigma-\beta-1)^{2}\right) d_{3}+\beta(\sigma-\beta-1)(\sigma+\beta+1)(\sigma+1)\right) d_{2}\right]}{e \omega(\sigma-1)(\sigma-\beta-1)(\sigma+\beta+1)} \\
& e_{6}=\frac{A_{2} \sigma(\sigma-\beta-1)\left[-2 e d_{2}+(\sigma+\beta+1) d_{3}\right]}{\left(\sigma^{2}-1\right)(\sigma+\beta+1)} \\
& \mathrm{e}=\sqrt{\frac{\beta(\sigma+\beta+1)(\sigma+1)}{\sigma-\beta-1}} \\
& A_{2}=\frac{-\omega-i(\sigma+1)}{2 e \sigma A} \\
& d_{1}=\omega\left(-\beta-1-\beta \sigma^{2}-e^{2} \sigma+e^{2} \beta-2 \sigma-\sigma^{2}+e^{2}-2 \sigma \beta\right) \\
& -i\left(e^{2} \beta \sigma+\omega^{2}+\sigma \omega^{2}+\beta \omega^{2}+\sigma \beta \omega^{2}-\sigma^{2} e^{2}+e^{2} \sigma\right) \\
& d_{2}=-\omega \sigma-\omega \sigma^{3}-2 \omega \sigma^{2}+\mathrm{i}\left(-\sigma^{2} \omega^{2}+e^{2} \sigma+e^{2} \sigma \beta-e^{2} \sigma^{2}-\sigma \omega^{2}\right) \\
& d_{3}=-\sigma^{2} e \omega-e \sigma \omega+i\left(2 e \sigma^{2}+e \sigma^{2} \beta+e \sigma \beta+e \sigma^{3}+e \sigma\right) \\
& \text { And } \\
& \mathrm{A}=\omega^{2}+3 \sigma+3 \sigma^{2}+\sigma \omega^{2}+\sigma^{3}+1+\beta+2 \beta \sigma+\beta \sigma^{2}+e^{2} \sigma-e^{2}-e^{2} \beta
\end{aligned}
$$


Where an over bar denotes complex conjugation. According to the center manifold theorem $W^{c}$ is tangent to $E^{c}=\operatorname{span}\{\mathrm{v}, \mathrm{w}\}$. Therefore, $W^{c}$ can be approximated for the two variables $\mathrm{v}, \mathrm{w}$ by equation

$$
\mathrm{u}=\mathrm{h}(\mathrm{v}, \mathrm{w})=\alpha v^{2}+\delta \mathrm{vw}+\gamma w^{2}+\mathrm{O}(3)
$$

Where $\mathrm{O}(3)$ denotes terms of order $v^{3}, v^{2} w, v w^{2}$ and $w^{3}$. With

$$
\dot{u}=\frac{\partial h}{\partial v} \dot{v}+\frac{\partial h}{\partial w} \dot{w}
$$

it follows together with eq(27) and eq(28), after comparison of the coefficients for $v^{2}$, vw and $w^{2}$ obtains:

$$
\alpha=\frac{a_{2}}{\sigma+\beta+1-2 i \omega}, \delta=\frac{a_{4}}{\sigma+\beta+1} \text { and } \gamma=\frac{\bar{a}_{2}}{\sigma+\beta+1+2 i \omega}
$$

Also

$$
\begin{aligned}
& \mathrm{u}=\mathrm{h}(\mathrm{v}, \mathrm{w})=\frac{a_{2}}{\sigma+\beta+1-2 i \omega} v^{2} \\
& +\frac{a_{4}}{\sigma+\beta+1} \mathrm{vw}+\frac{\bar{a}_{2}}{\sigma+\beta+1+2 i \omega} w^{2}+\mathrm{O}(3)
\end{aligned}
$$

In the same way according to theorem (3) one can approximate the center manifold up to any order.

After inserting $\mathrm{u}=\mathrm{h}(\mathrm{v}, \mathrm{w})$ to in the equations for $\mathrm{v}, \mathrm{w}$ in eq(27) obtains an approximated expression for the flow in the center manifold:

$$
\left[\begin{array}{l}
\dot{v} \\
\dot{w}
\end{array}\right]\left[\begin{array}{c}
\mathrm{i} \omega \mathrm{v} \\
\mathrm{i} \omega \mathrm{w}
\end{array}\right)=\left(\begin{array}{c}
e_{2} v^{2}+e_{3} w^{2}+e_{6} v w+\left(e_{4} v+e_{5} w\right) u \\
\bar{e}_{3} v^{2}+\bar{e}_{2} w^{2}+\bar{e}_{6} v w+\left(\bar{e}_{5} v+\bar{e}_{4} w\right) u
\end{array}\right)+\mathrm{O}(4) \ldots(31)
$$

In the second step we now simplify the expression for the flow in the center manifold by removing all of the redundant non linear terms. The simplest expression is the normal form which still contains all information about the qualitative behavior of the system of the bifurcation point. With a further linear coordinate transformation system (31) can be rewritten in to a form which only contains real numbers giving the so-called standard form. With

$$
\left[\begin{array}{l}
\mathrm{v} \\
\mathrm{w}
\end{array}\right)=\mathrm{T}\left(\begin{array}{l}
\zeta \\
\chi
\end{array}\right) \quad ; \mathrm{T}=\left(\begin{array}{cc}
1 & -\mathrm{i} \\
1 & \mathrm{i}
\end{array}\right)
$$

It follows 


$$
\begin{aligned}
& \dot{\zeta}=-\omega \chi+\frac{1}{2}\left(-e_{3}-\bar{e}_{3}-e_{2}-\bar{e}_{2}+e_{6}+\bar{e}_{6}\right) \chi^{2}+\frac{1}{2}\left(e_{3}+\bar{e}_{3}+e_{2}+\bar{e}_{2}+e_{6}+\bar{e}_{6}\right) \zeta^{2}+\mathrm{i} \\
& \left(e_{3}-\bar{e}_{3}-e_{2}+\bar{e}_{2}\right) \zeta \chi+\frac{1}{2}\left[\left(e_{4}+\bar{e}_{5}\right)\left(\frac{a_{2}}{\sigma+\beta+1-2 i \omega}+\frac{a_{4}}{\sigma+\beta+1}+\frac{\bar{a}_{2}}{\sigma+\beta+12 i \omega}\right)\right. \\
& \left.+\left(e_{5}+\bar{e}_{4}\right)\left(\frac{a_{2}}{\sigma+\beta+1-2 i \omega}+\frac{a_{4}}{\sigma+\beta+1}+\frac{\bar{a}_{2}}{\sigma+\beta+1+2 i \omega}\right)\right] \zeta^{3}+\frac{1}{2} \mathrm{i}\left[( e _ { 4 } + \overline { e } _ { 5 } ) \left(-\frac{3 a_{2}}{\sigma+\beta+1-2 i \omega}\right.\right. \\
& \left.\left.-\frac{a_{4}}{\sigma+\beta+1}+\frac{\bar{a}_{2}}{\sigma+\beta+1+2 i \omega}\right)+\left(e_{5}+\bar{e}_{4}\right)\left(-\frac{a_{2}}{\sigma+\beta+1-2 i \omega}+\frac{a_{4}}{\sigma+\beta+1}+\frac{\bar{a}_{2}}{\sigma+\beta+1+2 i \omega}\right)\right] \\
& +\frac{1}{2}\left[\left(e_{4}+\bar{e}_{5}\right)\left(-\frac{3 a_{2}}{\sigma+\beta+1-2 i \omega}+\frac{a_{4}}{\sigma+\beta+1}+\frac{\bar{a}_{2}}{\sigma+\beta+1+2 i \omega}\right)+\left(e_{5}+\bar{e}_{4}\right)\left(\frac{a_{2}}{\sigma+\beta+1-2 i \omega}\right.\right. \\
& +\frac{a_{4}}{\sigma+\beta+1}-\frac{3 \bar{a}_{2}}{\sigma+\beta+1+2 i \omega} \zeta \chi^{2}+\frac{1}{2} \mathrm{i}\left[( e _ { 4 } + \overline { e } _ { 5 } ) \left(\frac{a_{2}}{\sigma+\beta+1-2 i \omega}-\frac{a_{4}}{\sigma+\beta+1}\right.\right. \\
& \left.\left.+\frac{\bar{a}_{2}}{\sigma+\beta+1+2 i \omega}\right)\left(e_{5}+\bar{e}_{4}\right)\left(-\frac{a_{2}}{\sigma+\beta+1-2 i \omega}+\frac{a_{4}}{\sigma+\beta+1}-\frac{\bar{a}_{2}}{\sigma+\beta+1+2 i \omega}\right)\right] \chi^{3} \\
& \dot{\chi}=\omega \zeta-\frac{i}{2}\left(e_{3}-\bar{e}_{3}+e_{2}-\bar{e}_{2}-e_{6}+\bar{e}_{6}\right) \chi^{2}-\frac{i}{2}\left(-e_{3}+\bar{e}_{3}-e_{2}+\bar{e}_{2}-e_{6}+\bar{e}_{6}\right) \\
& \zeta^{2}+\left(-e_{3}-\bar{e}_{3}+e_{2}+\bar{e}_{2}\right) \zeta+\frac{i}{2}\left[\left(e_{4}-\bar{e}_{5}\right)\left(\frac{a_{2}}{\sigma+\beta+1-2 i \omega}+\frac{a_{4}}{\sigma+\beta+1}+\frac{\bar{a}_{2}}{\sigma+\beta+1+2 i \omega}\right)\right. \\
& \left.+\left(e_{5}-\bar{e}_{4}\right)\left(\frac{a_{2}}{\sigma+\beta+1-2 i \omega}+\frac{a_{4}}{\sigma+\beta+1}+\frac{\bar{a}_{2}}{\sigma+\beta+1+2 i \omega}\right)\right] \zeta^{3}-\frac{1}{2}\left[\left(e_{4}-\bar{e}_{5}\right)\right. \\
& \left(-\frac{3 a_{2}}{\sigma+\beta+1-2 i \omega} \frac{a_{4}}{\sigma+\beta+1}+\frac{\bar{a}_{2}}{\sigma+\beta+1+2 i \omega}+\left(e_{5}-\bar{e}_{4}\right)\left(\frac{a_{2}}{\sigma+\beta+1-2 i \omega}+\frac{a_{4}}{\sigma+\beta+1}\right.\right. \\
& \left.\left.+\frac{3 \bar{a}_{2}}{\sigma+\beta+1+2 i \omega}\right)\right]+\frac{i}{2}\left[\left(e_{4}-\bar{e}_{5}\right)\left(-\frac{3 a_{2}}{\sigma+\beta+1-2 i \omega}+\frac{a_{4}}{\sigma+\beta+1}+\frac{\bar{a}_{2}}{\sigma+\beta+1+2 i \omega}\right)\right. \\
& +\left(e_{5}-\bar{e}_{4}\right)\left(\frac{a_{2}}{\sigma+\beta+1-2 i \omega}+\frac{a_{4}}{\sigma+\beta+1}-\frac{3 \bar{a}_{2}}{\sigma+\beta+1+2 i \omega}\right) \zeta \chi^{2}-\frac{1}{2}\left[\left(e_{4}-\bar{e}_{5}\right)\right. \\
& \left(\frac{a_{2}}{\sigma+\beta+1-2 i \omega}-\frac{a_{4}}{\sigma+\beta+1}+\frac{\bar{a}_{2}}{\sigma+\beta+1+2 i \omega}\right)\left(e_{5}-\bar{e}_{4}\right)\left(-\frac{a_{2}}{\sigma+\beta+1-2 i \omega}+\frac{a_{4}}{\sigma+\beta+1}\right. \\
& \left.\left.-\frac{\bar{a}_{2}}{\sigma+\beta+1+2 i \omega}\right)\right] \chi^{3}
\end{aligned}
$$

Guckenheimer and Holmes have explicitly [5] shown that on the basis of the normal form theorem one finds a nonlinear coordinate transformation which transforms every system with the structure.

$$
\zeta=-\omega \chi+O(|\zeta|,|\chi|)
$$




$$
\chi \cdot=\omega \zeta+O(|\zeta|,|\chi|)
$$

to in the system

$$
\begin{aligned}
& \dot{u}=-\omega v+(\mathrm{au}-\mathrm{bv})\left(u^{2}+v^{2}\right)+\mathrm{O}(4) \\
& \dot{v}=\omega \mathrm{u}+(\mathrm{au}+\mathrm{bv})\left(u^{2}+v^{2}\right)+\mathrm{O}(4)
\end{aligned}
$$

This is expressed in polar coordinates as:

$$
\begin{aligned}
& \dot{\rho}=a \rho^{3} \\
& \dot{\theta}=\omega+b \rho^{2}
\end{aligned}
$$

It can be seen that the sign of determining the stability of the equilibrium point is at the Hopf bifurcation point. Guckenheimer and Holmes [5] carried out the procedure for calculating the coefficient a and gave the formula:

$$
\begin{aligned}
\mathrm{a}= & \frac{1}{16}\left(f_{x x x}+f_{x y y}+g_{x x y}+g_{y y y}+(1 / \omega)\left(f_{x y}\left(f_{x x}+f_{y y}\right)\right.\right. \\
& \left.\left.-g_{x y}\left(g_{x x}+g_{y y}\right)-f_{x x} g_{x x}+f_{y y} g_{y y}\right)\right)
\end{aligned}
$$

where $f_{x y}$ denotes $\frac{\partial^{2} f(0,0)}{\partial x \partial y}$, etc. And $\mathrm{f}, \mathrm{g}$ are the functions containing the nonlinear terms of equation (33)(for more detail see[5]). Also the coefficient $\mathrm{b}$ gives the formula:

$$
\begin{aligned}
\mathrm{b} & =\frac{1}{16}\left[g_{x x x}+g_{x y y}-f_{x x y}-f_{y y y}+\frac{1}{3 \omega}\left(5 \left(f_{x x} g_{x y}+f_{x y} g_{y y}-f_{x x} f_{y y}-f_{y y}^{2}\right.\right.\right. \\
& \left.\left.\left.-g_{x x} g_{y y}-g_{x x}^{2}\right)-2\left(f_{x x}^{2}+f_{x y}^{2}+g_{x y}^{2}+g_{y y}^{2}\right)+f_{y y} g_{x y}+f_{x y} g_{x x}\right)\right] \quad \ldots(37)
\end{aligned}
$$

Applying the equation $(36,37)$ to expression $(32)$ which has structure of (33) to obtain:

$$
\begin{aligned}
& a=\frac{1}{2}\left[\frac{a_{2} e_{5}}{\sigma+\beta+1-2 i \omega}+\frac{\bar{a}_{2} \bar{e}_{5}}{\sigma+\beta+1+2 i \omega}+\frac{a_{4}\left(e_{4}+\bar{e}_{4}\right)}{\sigma+\beta+1}+\frac{i}{\omega}\left(-e_{2} e_{6}+\bar{e}_{2} \bar{e}_{6}\right)\right] \\
& b=\frac{1}{2}\left[i\left[\frac{a_{2} e_{5}}{\sigma+\beta+1-2 i \omega}-\frac{\bar{a}_{2} \bar{e}_{5}}{\sigma+\beta+1+2 i \omega}+\frac{a_{4}\left(e_{4}-\bar{e}_{4}\right)}{\sigma+\beta+1}\right]+\frac{1}{3 \omega}\left[3\left(e_{2} e_{6}+\bar{e}_{2} \bar{e}_{6}\right)-4\left(e_{3} \bar{e}_{3}\right)-6\left(e_{6} \bar{e}_{6}\right)\right]\right]
\end{aligned}
$$

using Maple program to simplify eq(38) after substituting the values of a2,a4, e2,e3,e4,e5,e6, ā 2, $\mathrm{e}_{2}, \overline{\mathrm{e}}_{3}, \overline{\mathrm{e}}_{4}, \overline{\mathrm{e}}_{5}, \mathrm{e}_{6}, \mathrm{~A}, \mathrm{~A}_{2}$, e and $\omega$ obtains

$$
\begin{aligned}
& a=\frac{\sigma \beta(\sigma-\beta-1)^{2}\left(\beta^{4}+2 \sigma \beta^{4}+6 \beta^{3}-20 \sigma^{2} \beta^{2}+12 \beta^{2}-\sigma \beta^{2}+18 \beta \sigma^{3}-2 \beta \sigma^{2}-10 \sigma \beta+10 \beta-9 \sigma^{3}-20 \sigma^{3}-10 \sigma^{2}+4 \sigma+3\right)}{2(\sigma+\beta+1)\left(\sigma^{2}-1\right)\left(\sigma^{3}+3 \sigma^{2} \beta+\sigma^{2}-\sigma \beta^{2}-\sigma-1-\beta^{3}-3 \beta^{2}-3 \beta\right)\left(\sigma^{3}+\sigma^{2}+9 \beta \sigma^{2}-\sigma+6 \sigma \beta-\sigma \beta^{2}-1-3 \beta-3 \beta^{2}-\beta^{3}\right)} \\
& b=\frac{\sqrt{2 \sigma(\sigma-\beta-1)^{3}}}{6(\sigma-1)(\sigma+\beta+1) \sqrt{\beta(\sigma+1)^{3}}\left(\sigma^{3}+9 \beta \sigma^{2}+\sigma^{2}+6 \sigma \beta-\sigma-\sigma \beta^{2}-\beta^{3}-1-3 \beta^{2}-3 \beta\right)\left(\sigma^{3}+3 \beta \sigma^{2}+\sigma^{2}-\sigma \beta^{2}-\sigma-1-3 \beta^{2}-3 \beta-\beta^{3}\right)}[
\end{aligned}
$$


$26 \beta \sigma^{6}+2 \sigma-46 \beta^{4} \sigma^{2}+13 \beta^{5} \sigma^{2}+8 \beta^{6} \sigma-111 \beta^{4} \sigma^{3}+4 \sigma^{6}+9 \beta^{3} \sigma^{4}-5 \beta^{2}-\beta-79 \beta^{3} \sigma^{2}+2 \sigma^{7}-5 \beta^{5}-2 \sigma^{5}+91 \beta^{4} \sigma$

$+113 \beta^{2} \sigma^{4}+44 \beta^{5} \sigma-8 \sigma^{4}-2 \sigma^{3}+12 \beta \sigma^{2}+4 \sigma^{2}-10 \beta^{3}-156 \sigma^{3} \beta^{2}-50 \beta \sigma^{3}+17 \sigma \beta+55 \sigma \beta^{2}-60 \sigma^{2} \beta^{2}-37 \sigma^{4} \beta$

$\left.+95 \beta^{3} \sigma+33 \sigma^{5} \beta-175 \sigma^{3} \beta^{3}-10 \beta^{4}-\beta^{6}+149 \sigma^{5} \beta^{2}\right]$

Since $\sigma>\beta+1$, we can write $\sigma=\beta+1+c, \mathrm{c}>0$ after substituting $\sigma=\beta+1+c$ in eq (31), Maple program tells us $\mathrm{a}>0$ and $\mathrm{b}<0$ for all $\sigma, \beta$ defined above. It follows from a $>0$ that the equilibrium point $A_{+}$at the bifurcation point is unstable. Since $\omega>0$ and $b<0$ it can be seen from (35) that there is a critical value $\rho=\rho^{*}$ where the direction of rotation changes. Since $\mathrm{a}>0$ according to the Hopf bifurcation theorem, the system (1) has unstable limit cycle (sub critical Hopf bifurcation). If we take $\sigma=10, \beta=\frac{8}{3}$ as example then $\mathrm{a}=0.004580131420$ and $\mathrm{b}=-0.05344960599$ since a $>0$ system (1) has unstable limit cycle see, figure (2).

From eq(9) because the $\dot{\rho}$ is separated,so.

$$
\rho=\sqrt{\frac{d \mu}{\left(a+\frac{d \mu}{r_{0}}\right) e^{(-2 d \mu t)}}}
$$

Since the parameters a and d are known from (eq (39),eq (25)) we have an up to third order approximate expression for the radius of the limit cycle and the dynamics with which the limit cycle will be reached depending on the parameter $\mu$.

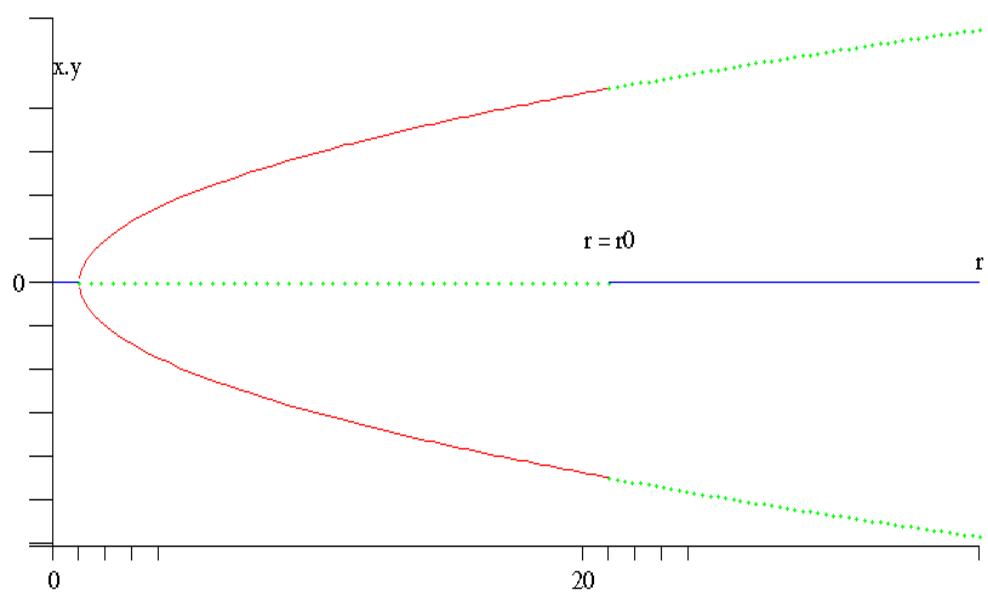


(a)

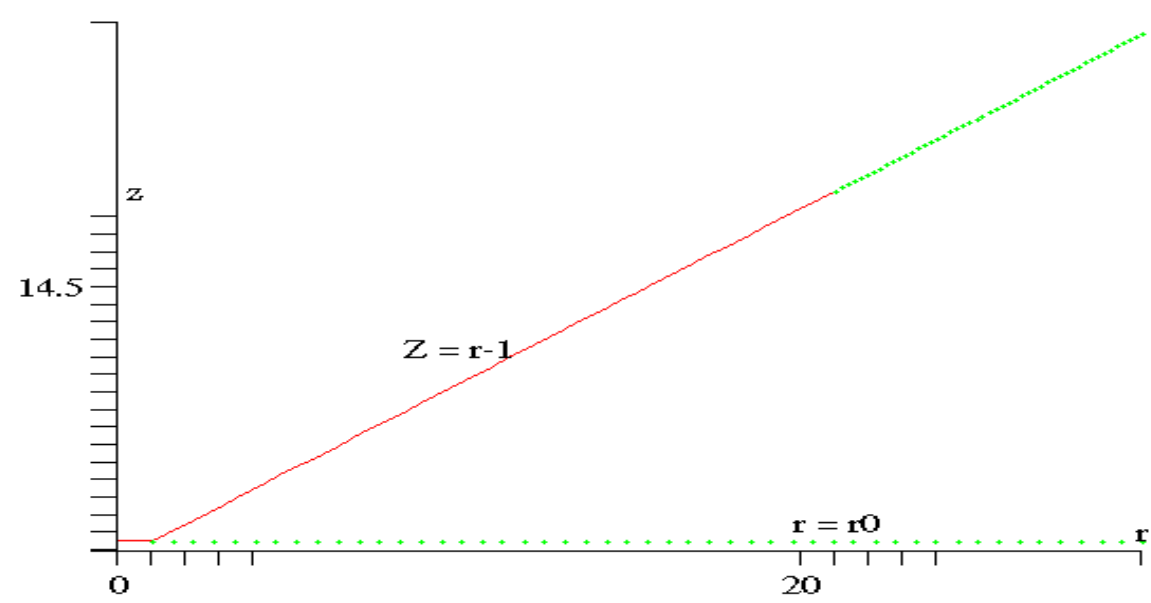

(b)

Figure (1): Equilibrium bifurcation diagrams of Lorenz system dependence on $\mathrm{r}$ of: (a) x,y; (b) z. The solid curves depict stable behavior and the doted curves depict unstable behavior

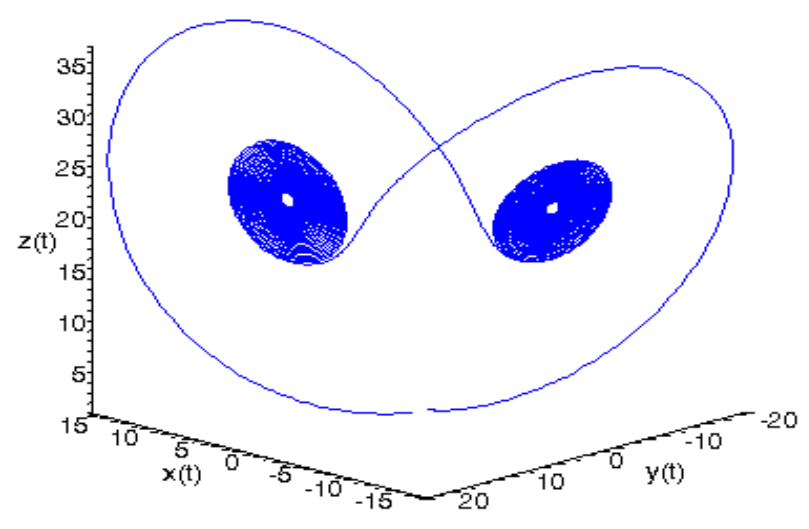

Figure(2.a)phase portraite for system(1)when $\sigma=10, \beta=\frac{8}{3}$ and $r=22$ 


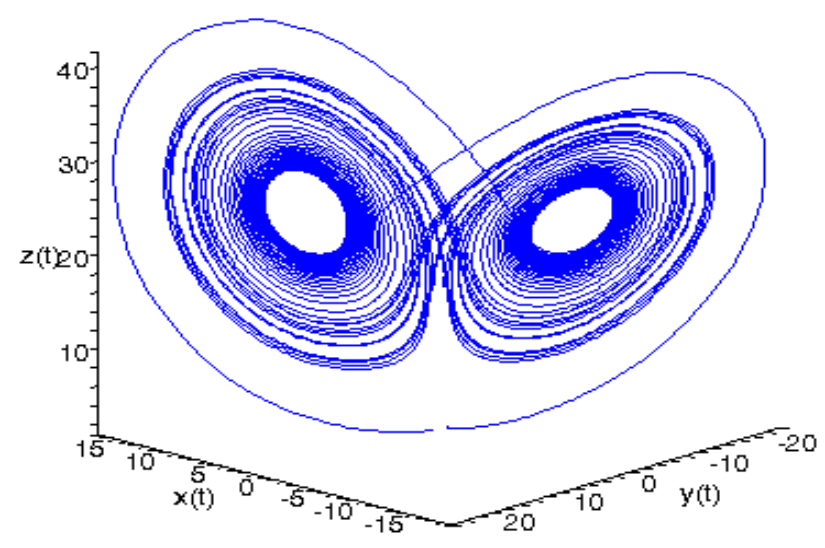

Figure (2.b): phase portrait for system (1) when $\sigma=10, \beta=\frac{8}{3}$ and $r=\frac{470}{19}$.

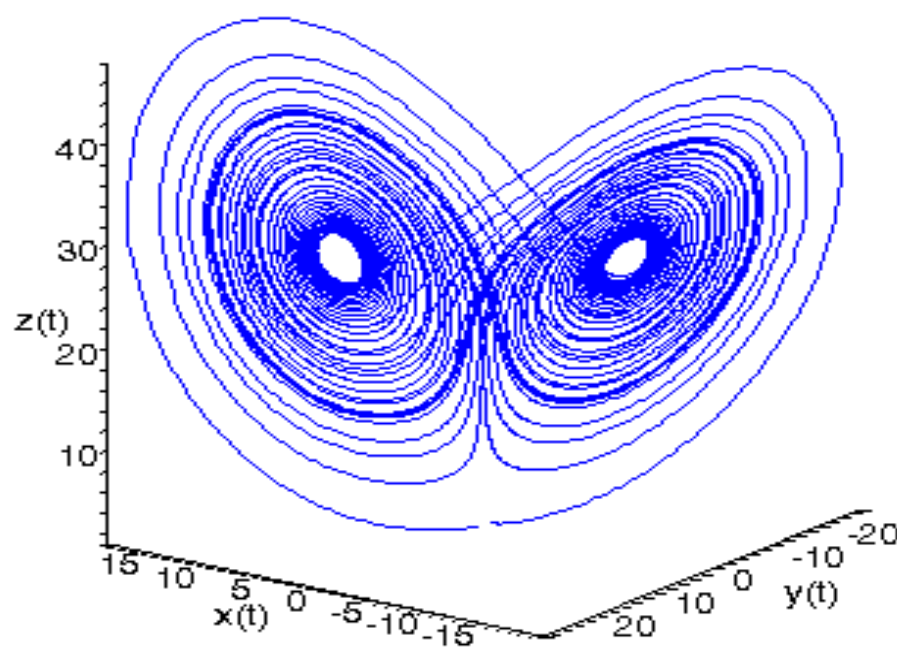

Figure (2.c): phase portrait for system (1) when $\sigma=10, \beta=\frac{8}{3}$ and $r=28$. 


\section{Conclusions}

In this paper we began to study the Lorenz system. Some insights on stability and bifurcation are obtained. The system posses a Hopf bifurcation and, in particular the case which is analyzed obtained that bifurcation is subcritical (unstable limit cycle). Analyzing the Hopf bifurcation we show that the arising unstable limit cycle is near a bifurcation point. It means that, for the Lorenz system, the appearance and location of limit cycles in vicinity at the equilibrium only occurs at very specific values of the parameters defining the equations. 


\section{REFRENCES}

[1] Ahmad,.N.M, "The Periodic Solutions for Controlled Biological Systems", Msc .Thesis,College of Siences ,University of Baghdad, (1992).

[2] Carr,J., "Application of Center Manifold Theory”, Springer-verlag, New York (1981).

[3] Char, B.W., "Maple 7 Learning Guide", Waterloo maple Inc., (2001).

[4] David,B and Mylan,R "Mathematical Computing An Introduction to Programming Using Maple”, Springer- verlag New York, (2002).

[5] Guckenheimer.J and Holmes.P, "Nonlinear oscillations, Dynamical systems, and Bifurcations of vector Fields", Springer, New York, (2002).

[6] Lorenz, E.N, "Deterministic Non-Periodic Flow”, J. Atmos. Sci, vol 20, (1963), 130-141.

[7] Lynch, S., "Dynamical Systems With Applications Using Maple", Birkhauser, Boston, (2001).

[8] Marsden, J.E and McCracken, M.,"The Hopf Bifurcation and its Application", Springer-verlag.,NY, (1978).

[9] Palacian,J. and Yanguas.p, "Periodic Orbits of the Lorenz System through Perturbation Theory",.International Journal of Bifurcation and Chaos, vol.11, No.10 (2001), 2559-2566

[10] Pushpavanam ,S.,"Mathematical Methods in Chemical Engineering" , prentice, Hall private.Limited, New Delhi, (2005).

[11] Qian,H., "Deterministic and stochastic Dynamical System", Springer, (2003)

[12] Rao,M.R., “Ordinary Differential Equation”, Affiliated east-west press , New Delhi-Madras, (1980).

[13] Stephen,J.Merrill, "A Model of the Stimulation of B-cells by Replicating Antigen-II”.,Mathematical Biosciences, 41: (1978) 143155.

[14] Sparrow, C. "The Lorenz Equation, Bifurcation, Chaos and Strange Attractors", Springer- Verlag ,New York, (1982).

[15] Teschl,G., "Ordinary Differential Equation and Dynamical Systems", typeset by Ams-latex and Makeindex,(2004). 
[16] Yu,P. "Computation of Normal Form via a Perturbation Technique" Journal of Sound andVibration 211(1), (1988) ,19-38.

[17] Yu,P., "Simplest Normal forms of Hopf and Generalized Hopf Bifurcations", International Journal of Bifurcation and Chaos, Vol.9, N.10 (1999) 1917-1923.

[18] Yu,P. "A matching Pursuit Technique for Computing the Simplest Normal Form of Vecter Field", Jornal of Symbolic Computation, 35, (2003) 591-615. 